Louisiana State University

LSU Digital Commons

Faculty Publications

Department of Mathematics

7-1-2019

\title{
On average values of time-varying delays and a new representation of systems with time-varying delays
}

Frederic Mazenc

Laboratoire des Signaux et Systèmes

Michael Malisoff

Louisiana State University

Follow this and additional works at: https://digitalcommons.Isu.edu/mathematics_pubs

\section{Recommended Citation}

Mazenc, F., \& Malisoff, M. (2019). On average values of time-varying delays and a new representation of systems with time-varying delays. Proceedings of the American Control Conference, 2019-July, 3714-3718. https://doi.org/10.23919/acc.2019.8814964

This Conference Proceeding is brought to you for free and open access by the Department of Mathematics at LSU Digital Commons. It has been accepted for inclusion in Faculty Publications by an authorized administrator of LSU Digital Commons. For more information, please contact ir@lsu.edu. 


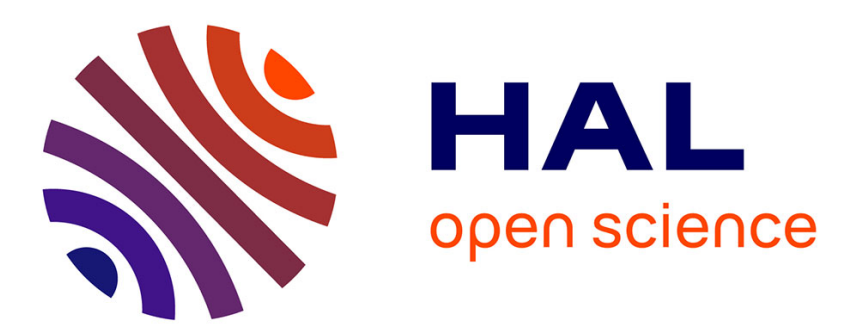

\section{On Average Values of Time-Varying Delays and a New Representation of Systems with Time-Varying Delays}

Frédéric Mazenc, Michael Malisoff

\section{To cite this version:}

Frédéric Mazenc, Michael Malisoff. On Average Values of Time-Varying Delays and a New Representation of Systems with Time-Varying Delays. ACC 2019 - American Control Conference, Jul 2019, Philadelphia, United States. hal-02268094

\section{HAL Id: hal-02268094 \\ https://hal.inria.fr/hal-02268094}

Submitted on 20 Aug 2019

HAL is a multi-disciplinary open access archive for the deposit and dissemination of scientific research documents, whether they are published or not. The documents may come from teaching and research institutions in France or abroad, or from public or private research centers.
L'archive ouverte pluridisciplinaire HAL, est destinée au dépôt et à la diffusion de documents scientifiques de niveau recherche, publiés ou non, émanant des établissements d'enseignement et de recherche français ou étrangers, des laboratoires publics ou privés. 


\section{On Average Values of Time-Varying Delays and a New Representation of Systems with Time-Varying Delays}

\author{
Frederic Mazenc
}

\author{
Michael Malisoff
}

\begin{abstract}
We show by a counterexample that the asymptotic stability of a system with a pointwise periodic time-varying delay cannot be deduced from the average value of the delay. We use this counterexample to motivate our new representation of systems with time-varying delays, which we use to develop a new state feedback stabilization method.

Index Terms - time-varying, delay, stability, averaging
\end{abstract}

\section{INTRODUCTION}

Averaging is a very successful technique that was developed in many contributions (including [5], [7], [9], and [15]) and has been applied to many engineering problems [3], [6]. On the other hand, many systems admit time-varying delays, so several contributions are devoted to systems with timevarying delays, including [2], [12], [13], [14], [16], [17], and [18]. For instance, sampling can be represented as a timevarying delay. Time-varying delays increase the difficulty of analyzing the stability of, or stabilizing, control systems. Fundamental approaches that are available include classical LMIs or prediction based control. They only apply under some conditions, which basically imply that the variations of the delay can be neglected.

A stability technique based on approximation of timevarying delays is proposed in [12]. The work [12] is a notable step in the topic of systems with time-varying delays because it can handle delays which are too big to be neglected, and it uses an original approach that introduces a comparison system with a distributed delay. However, the limitation of [12] is the fact that the kernel of the distributed term of the comparison system contains a function which is not given by an explicit formula and then stability results cannot be derived when the delay is not rapidly varying. Thus, when the time-varying part of the delay has big variations or is discontinuous and not small enough to be neglectable, then stability and stabilization problems are still open.

The previous comments motivate the following question: When a system has a time-varying periodic delay, can we use the average value of the delay to infer the stability properties of the system? For instance, one may wonder whether

$$
\dot{X}(t)=-X(t-\tau(v t))
$$

with its state $X$ valued in $\mathbb{R}$ is exponentially stable when $\tau:[0,+\infty) \rightarrow[0,+\infty)$ is periodic of period $T$ and such

Malisoff is with the Department of Mathematics, Louisiana State University, Baton Rouge, LA 70803-4918, USA, malisoff@lsu.edu.

Malisoff was supported by NSF Grant 1711299 .

Mazenc is with EPI DISCO INRIA-Saclay, Laboratoire des Signaux et Systèmes (L2S, UMR CNRS 8506), CNRS, CentraleSupélec, Université Paris-Sud, 3 rue Joliot Curie, 91192, Gif-sur-Yvette, France, frederic.mazenc@12s.centralesupelec.fr. that

$$
\frac{1}{T} \int_{0}^{T} \tau(m) \mathrm{d} m=1
$$

and the constant $v>0$ is large, because the origin of the system $\dot{X}(t)=-X(t-1)$ is exponentially stable (by [4, Example 2.3]). Answering this question is challenging because, as shown in [8], a time-varying delay can be deceitful, insofar that [8] proves that $\dot{X}(t)=-X(t)-1.5 X(t-\eta)$ with $X$ valued in $\mathbb{R}$ is exponentially stable for each constant $\eta \in[0,2.05]$, but that there is a piecewise continuous $h$ such that $h(t) \in[0,2.05]$ for all $t \geq 0$ and such that $\dot{X}(t)=-X(t)-1.5 X(t-h(t))$ has an unbounded solution.

In this work, we establish through an example that when a system has a pointwise periodic time-varying delay, it can be unstable even when it is rapidly varying and when it is stable when the delay is replaced by its average value. This fact is in sharp contrast with the averaging results available for linear systems with a time-varying periodic vector field.

To apply techniques available for time-varying systems with constant pointwise delays, we also propose a new representation of systems with time-varying delays which can be decomposed into a switched delay plus a small time-varying term. It involves a sum of terms with constant delays and time-varying coefficients plus an extra small term. We believe that this new representation is a valuable contribution of our paper because it sheds light on the fundamental mechanisms of stabilization and destabilization inherent in time-varying delays and is a bridge between two distinct classical types of systems, namely, systems with time-varying delays and timevarying systems with several constant delays. To the best of our knowledge, this representation is new, but it shares features with the representation of time-varying systems introduced in [11, Section 3.1] (which did not use averaging). In the last part of our work, we use our new representation to extend the reduction model approach (studied for instance in [9]) to a new family of systems with time-varying delays, which can be discontinuous and whose derivative, when defined, can be larger than 1 . This extension is completely different from the approach of [1]. The results we propose here are well adapted to systems with switched delays. For this family of systems, we believe that the reduction model or predictive approaches had not been developed.

The notation will be simplified whenever no confusion can arise from the context. The dimensions of our Euclidean spaces are arbitrary unless otherwise noted. For a constant $T>0, C_{\text {in }}$ denotes the set of all continuous functions $\phi$ : $[-T, 0] \rightarrow \mathbb{R}^{a}$, which we call the set of all initial functions. We define $\Xi_{t} \in C_{\text {in }}$ by $\Xi_{t}(s)=\Xi(t+s)$ for all $\Xi, s \leq 0$, 
and $t \geq 0$ for which the equality is defined. We let $\mid$. denote the usual Euclidean norm of vectors and the induced matrix norm, and $\mathbb{N}=\{1,2, \ldots\}$.

\section{PRELIMINARY RESUltS}

Consider the system

$$
\dot{x}(t)=f(t, x(t), x(t-\tau(t)))
$$

with $x$ valued in $\mathbb{R}^{n}$ and with initial conditions in $C_{\text {in }}$, where $f$ is a nonlinear locally Lipschitz function and where the delay $\tau(t)$ is nonnegative valued and piecewise continuous and bounded from above by a known constant $\bar{\tau}$. In our first lemma, we will assume:

Assumption 1: The system (3) is forward complete. Also, there exist a functional $\mathcal{S}: C_{\text {in }} \rightarrow[0, \infty)$, constants $s_{i}>0$ for $i=1$ to 4 , and a constant $T \geq \bar{\tau}$ such that for all $\phi \in C_{\text {in }}$, we have

$$
s_{1}|\phi(0)|^{2} \leq \mathcal{S}(\phi) \leq s_{2} \sup _{m \in[-T, 0]}|\phi(m)|^{2}
$$

for all $t \geq 0$, and such that the time derivative of $\mathcal{S}$ along all solutions of (3) is continuous and satisfies

$$
\dot{\mathcal{S}}(t) \geq s_{3} \mathcal{S}\left(x_{t}\right)-s_{4} \sup _{m \in[\max \{0, t-T\}, t]} \mathcal{S}\left(x_{m}\right)
$$

for all $t \geq 0$ and $s_{3}>s_{4}$.

We prove the following:

Lemma 1: If the system (3) satisfies Assumption 1, then this system admits an unbounded solution.

Proof: Consider any solution $x(t)$ of the system (3) with a constant initial condition $\phi(t)=\phi_{c} \neq 0$ for all $t \in$ $[-T, 0]$. We first prove that for this solution, $\dot{\mathcal{S}}(t)>0$ for all $t \geq 0$. To this end, note that since $\phi(t)=\phi_{c} \neq 0$ for all $t \in[-T, 0]$, it follows from (4) that $\mathcal{S}\left(x_{0}\right) \geq s_{1}\left|\phi_{c}\right|^{2}>0$. Since $s_{3}>s_{4}$, our condition (5) therefore gives $\dot{\mathcal{S}}(0) \geq$ $s_{3} \mathcal{S}\left(x_{0}\right)-s_{4} \mathcal{S}\left(x_{0}\right)>0$. Next, let us proceed by contradiction to prove that $\dot{\mathcal{S}}(t)>0$ for all $t \geq 0$. Let us suppose that there is $t_{c}>0$ such that for all $t \in\left[0, t_{c}\right), \dot{\mathcal{S}}(t)>0$ and $\dot{\mathcal{S}}\left(t_{c}\right)=0$.

Since for all $t \in\left[0, t_{c}\right), \dot{\mathcal{S}}(t)>0$, it follows that $\mathcal{S}\left(x_{t}\right)$ is nondecreasing over $\left[0, t_{c}\right]$. Consequently, $\mathcal{S}\left(x_{t_{c}}\right)=$ $\sup _{m \in\left[\max \left\{0, t_{c}-T\right\}, t_{c}\right]} \mathcal{S}\left(x_{m}\right) \geq \mathcal{S}\left(x_{0}\right)>0$. It follows that $\dot{\mathcal{S}}\left(t_{c}\right) \geq s_{3} \mathcal{S}\left(x_{t_{c}}\right)-s_{4} \mathcal{S}\left(x_{t_{c}}\right)>0$. This yields a contradiction with the definition of $t_{c}$. We conclude that for all $t \geq 0, \dot{\mathcal{S}}(t)>0$. Therefore, $\mathcal{S}\left(x_{t}\right)$ is nondecreasing over $[0,+\infty)$. Hence, for all $t \geq 0$, we have $\sup _{m \in[\max \{0, t-T\}, t]} \mathcal{S}\left(x_{m}\right)=\mathcal{S}\left(x_{t}\right)$. This equality, in combination with (5), gives $\dot{\mathcal{S}}(t) \geq\left(s_{3}-s_{4}\right) \mathcal{S}\left(x_{t}\right)$ for all $t \geq 0$. It follows that $\lim _{t \rightarrow+\infty} \mathcal{S}\left(x_{t}\right)=+\infty$, since $s_{3}>s_{4}$. We deduce from (4) that (3) admits an unbounded solution.

Remark 1: Observe that the case where the system (3) is not forward complete is trivial in the context of Lemma 1 because then the system admits an unbounded solution.

The formulas in the next lemma can be deduced easily by writing (6) in the form $\dot{g}=A g+B u$, then exponentiating the matrix $A$ to obtain a rotation matrix, and then applying variation of parameters, so we omit its proof:
Lemma 2: Consider the system

$$
\left\{\begin{array}{l}
\dot{g}_{1}(t)=g_{2}(t) \\
\dot{g}_{2}(t)=-g_{1}(t)+u(t)
\end{array}\right.
$$

with $g_{i}$ and $u$ valued in $\mathbb{R}$ and $u$ being continuous. Then,

$$
\begin{aligned}
g_{1}\left(t-t_{*}\right)= & \cos \left(t_{*}\right) g_{1}(t)-\sin \left(t_{*}\right) g_{2}(t) \\
& -\int_{t-t_{*}}^{t} \sin \left(t-m-t_{*}\right) u(m) \mathrm{d} m \\
g_{2}\left(t-t_{*}\right)= & \sin \left(t_{*}\right) g_{1}(t)+\cos \left(t_{*}\right) g_{2}(t) \\
& -\int_{t-t_{*}}^{t} \cos \left(t-m-t_{*}\right) u(m) \mathrm{d} m
\end{aligned}
$$

hold for all $t \in \mathbb{R}$ and all constants $t_{*}>0$.

In Appendix A, we prove:

Lemma 3: There is a constant $\underline{\epsilon} \in(0,1)$ such that for all $\epsilon \in(0, \underline{\epsilon}]$ the system

$$
\left\{\begin{array}{l}
\dot{y}_{1}(t)=y_{2}(t) \\
\dot{y}_{2}(t)=-y_{1}(t)+\epsilon y_{1}\left(t-\frac{3 \pi}{4}\right)
\end{array}\right.
$$

is globally exponentially stable to the origin.

\section{KEY EXAMPLE}

In this section, we use a switched system to establish a key result because it leads to a simple analysis, but a similar result can be proved with a $C^{1}$ time-varying delay.

Let $\tau_{0}=0$ and $\tau_{1}=\frac{3 \pi}{2}$ and $\nu>0$ be a constant. Consider

$$
\left\{\begin{array}{l}
\dot{x}_{1}(t)=x_{2}(t) \\
\dot{x}_{2}(t)=-x_{1}(t)+\epsilon x_{1}\left(t-\tau_{\sigma(t)}\right)
\end{array}\right.
$$

with $x_{i}$ valued in $\mathbb{R}$ for $i=1,2$, where $\epsilon \in(0, \underline{\epsilon})$ is a constant and $\underline{\epsilon}$ is from Lemma 3, and where $\sigma$ is periodic of period $2 \nu$ and satisfies (i) $\sigma(t)=0$ for all $t \in[0, \nu)$ and (ii) $\sigma(t)=1$ for all $t \in[\nu, 2 \nu)$. By the definition of $\sigma$, the function $\tau_{\sigma(t)}$ is periodic of period $2 \nu$ and its average value is $\frac{\tau_{0}+\tau_{1}}{2}=\frac{3 \pi}{4}$. Thus, if we replace $\tau_{\sigma(t)}$ by its average value in (9), then we obtain (8), which is exponentially stable.

However, we prove in Appendix B that there is a constant $\bar{\nu}>0$ such that (9) admits an unbounded solution when $\max \{\nu, \epsilon\}<\bar{\nu}$, using the fact that (9) has the representation

$$
\left\{\begin{aligned}
\dot{x}_{1}(t)= & x_{2}(t) \\
\dot{x}_{2}(t)= & -x_{1}(t)+\epsilon[1-\sigma(t)] x_{1}(t) \\
& +\epsilon \sigma(t) x_{1}\left(t-\frac{3 \pi}{2}\right)
\end{aligned}\right.
$$

as a time-varying system that is periodic of period $2 \nu$, with a constant delay. Thus, the average value of the delay $\tau_{\sigma(t)}$ does not indicate whether the system (9) is exponentially unstable or not, however fast the switches are.

\section{Time-VARYing Delay: NeW RePRESEntation}

The previous section suggests that systems with a switched delay can be represented as time-varying systems with several constant delays. We now describe this new representation in the general context of linear systems with time-varying delays, and we use this representation to solve a stabilization problem. Throughout the remaining part of the paper, we consider systems with time-varying delays $\tau$ that satisfy:

Assumption 2: The function $\tau:[0, \infty) \rightarrow[0, \infty)$ is piecewise continuous, periodic of some constant period $T>$ 0 , and bounded above by some finite constant $\bar{\tau}>0$. 
We also use the following notation. Let $q \in \mathbb{N}$, with $q \geq 2$, and set $\nu=\frac{T}{q}$. We set $t_{k}=k \nu$ for all integers $k \geq 0$. We define the switching signal $\sigma:[0,+\infty) \rightarrow\{0, \ldots, q-1\}$ as follows: $\sigma$ is periodic of period $T$ and $\sigma(t)=i$ for all $t \in[i \nu,(i+1) \nu)$ and all $i \in\{0, \ldots, q-1\}$. For all $i \in$ $\{0, \ldots, q-1\}$, we define the functions $\beta_{i}$ as follows: $\beta_{i}$ is periodic of period $T$ and satisfies (i) $\beta_{i}(t)=1$ for all $t \in$ $[i \nu,(i+1) \nu)$ and (ii) $\beta_{i}(t)=0$ for all $t \in[0, T)$ that are not in $[i \nu,(i+1) \nu)$. We introduce $q$ constants $h_{j} \geq 0$ for $j \in\{0, \ldots, q-1\}$ and define $r$ by $r(t)=\tau(t)-h_{\sigma(t)}$ for all $t \geq 0$. Let $\bar{r} \geq 0$ be a constant such that $|r(t)| \leq \bar{r}$ for all $t \geq 0$. Since $\tau$ is bounded, such an $\bar{r}$ exists.

Consider the system

$$
\dot{x}(t)=A x(t)+B u(t-\tau(t))
$$

where $x$ is valued in $\mathbb{R}^{n}$, the control $u$ is valued in $\mathbb{R}^{p}$, and $\tau$ satisfies Assumption 2. Since $\tau(t)=h_{\sigma(t)}+r(t)$ for all $t \geq 0$, the system (11) can be written as

$$
\begin{aligned}
\dot{x}(t)= & A x(t)+B u\left(t-h_{\sigma(t)}\right) \\
& +B\left[u\left(t-h_{\sigma(t)}-r(t)\right)-u\left(t-h_{\sigma(t)}\right)\right] .
\end{aligned}
$$

Then the definitions above yield the representation

$$
\begin{aligned}
\dot{x}(t)= & A x(t)+\sum_{i=0}^{q-1} \beta_{i}(t) B u\left(t-h_{i}\right) \\
& +B\left[u\left(t-h_{\sigma(t)}-r(t)\right)-u\left(t-h_{\sigma(t)}\right)\right] .
\end{aligned}
$$

A crucial question is how to choose the constants $h_{i}$. By increasing the number of constants $h_{i}$, one can decrease $\bar{r}$, which is desirable because we will treat the delay $r(t)$ as a disturbance in a later section. However, increasing the number of $h_{i}$ 's may increase the complexity of the problem one aims to solve. Thus the choice of the $h_{i}$ 's is a delicate issue. Note that (13) has several constant delays and a timevarying delay, while (11) has only one time-varying delay.

Control problems for the family of systems (11) have been studied in many contributions. However, our results are novel because of our mild Assumption 2, which does not require the condition $\dot{\tau}(t)<1$ when $\tau$ is differentiable. We assume:

Assumption 3: There is a continuous function $K$ that is bounded by a constant $\bar{K} \geq 0$ such that

$$
\begin{aligned}
\dot{\Theta}(t)= & H(t) \Theta(t)+B[K(t-\tau(t)) \Theta(t-\tau(t)) \\
& \left.-K\left(t-h_{\sigma(t)}\right) \Theta\left(t-h_{\sigma(t)}\right)\right], \text { where } \\
H(t)= & A+\sum_{i=0}^{q-1} e^{-A h_{i}} B \beta_{i}\left(t+h_{i}\right) K(t)
\end{aligned}
$$

is uniformly globally uniformly exponentially stable to 0 . $\square$

By a Lyapunov approach, one can easily prove that Assumption 3 holds if $\dot{\Theta}=H(t) \Theta(t)$ is uniformly exponentially stable to 0 and $\bar{r}>0$ is sufficiently small. We prove:

Theorem 1: Let the system (11) satisfy Assumption 3. Then the origin of (11) in closed-loop with

$$
u(t)=K(t)\left[x(t)+\sum_{i=0}^{q-1} \zeta_{i}(t)\right]
$$

and with the choices

$$
\zeta_{i}(t)=\int_{t-h_{i}}^{t} e^{A\left(t-s-h_{i}\right)} B \beta_{i}\left(s+h_{i}\right) u(s) \mathrm{d} s
$$

for all $i$ is uniformly globally uniformly exponentially stable.

Proof: We apply the reduction model approach to the system (13). Elementary calculations give

$$
\begin{aligned}
\dot{\zeta}_{i}(t)= & A \zeta_{i}(t)+e^{-A h_{i}} B \beta_{i}\left(t+h_{i}\right) u(t) \\
& -B \beta_{i}(t) u\left(t-h_{i}\right)
\end{aligned}
$$

for all $t \geq 0$. We next use the operator

$$
\lambda(t)=x(t)+\sum_{i=0}^{q-1} \zeta_{i}(t)
$$

By combining (13) with (17), we obtain

$$
\begin{aligned}
\dot{\lambda}(t)= & A \lambda(t)+\sum_{i=0}^{q-1} e^{-A h_{i}} B \beta_{i}\left(t+h_{i}\right) u(t) \\
& +B\left[u\left(t-h_{\sigma(t)}-r(t)\right)-u\left(t-h_{\sigma(t)}\right)\right] .
\end{aligned}
$$

Selecting the control law (15), we obtain

$$
\begin{aligned}
\dot{\lambda}(t)= & H(t) \lambda(t)+B[K(t-\tau(t)) \lambda(t-\tau(t)) \\
& \left.-K\left(t-h_{\sigma(t)}\right) \lambda\left(t-h_{\sigma(t)}\right)\right]
\end{aligned}
$$

where $H$ is from (14). By Assumption 3, it follows that $\lambda(t)$ is solution of a uniformly exponentially stable system. Since $u(t)=K(t) \lambda(t)$, it follows from (18) and our formula (16) for the $\zeta_{i}$ that the $x$ system is exponentially stable too.

\section{ILLUSTRATION}

Consider the one-dimensional system

$$
\dot{x}(t)=\frac{1}{8} x(t)+u(t-\tau(t))
$$

for any $\tau$ satisfying our Assumption 2. We apply Theorem 1 to find a feedback $u$ ensuring the global exponential stability of (21) when $T=2 \pi, q=4, \nu=\frac{\pi}{2}, h_{0}=0, h_{1}=h_{3}=\nu$, and $h_{2}=2 \nu$, by choosing the constant $\bar{r}>0$ small enough.

With the notation of Section IV with $A=\frac{1}{8}$ and $B=1$, we have

$$
\begin{aligned}
H(t)= & \frac{1}{8}+\left(\beta_{0}(t)+e^{-\frac{\nu}{8}}\left[\beta_{1}(t+\nu)\right.\right. \\
& \left.\left.+\beta_{3}(t+\nu)\right]+e^{-\frac{2 \nu}{8}} \beta_{2}(t+2 \nu)\right) K(t) .
\end{aligned}
$$

We choose $K(t)=-K_{*}$, where $K_{*}>0$ is any constant. Then $H$ has period $T$, so for all $t \geq T$, we obtain the average

$$
\begin{aligned}
H_{\star} & =\frac{1}{T} \int_{t-T}^{t} H(m) \mathrm{d} m=\frac{1}{T} \int_{0}^{T} H(m) \mathrm{d} m \\
& =\frac{1}{8}-\frac{K_{*}}{q}\left(1+2 e^{-\nu / 8}+e^{-\nu / 4}\right),
\end{aligned}
$$

since $\int_{0}^{T} \beta_{i}(\ell) \mathrm{d} \ell=\nu=T / q$ for all $i$.

Next, consider the system

$\dot{\lambda}(t)=H(t) \lambda(t)+K_{*}\left[-\lambda(t-\tau(t))+\lambda\left(t-h_{\sigma(t)}\right)\right]$

which corresponds to (14) from Assumption 3. Using the Fundamental Theorem of Calculus, we can rewrite (23) as

$$
\begin{aligned}
& \dot{\lambda}(t)=H(t) \lambda(t)+K_{*} \int_{t-h_{\sigma(t)}-r(t)}^{t-h_{\sigma(t)}} \dot{\lambda}(m) \mathrm{d} m \\
& =H(t) \lambda(t)+K_{*} \int_{t-h_{\sigma(t)}-r(t)}^{t-h_{(t)}}[H(m) \lambda(m) \\
& \left.+K_{*}\left[\lambda\left(m-h_{\sigma(m)}\right)-\lambda(m-\tau(m))\right]\right] \mathrm{d} m,
\end{aligned}
$$

where (24) and the equalities and inequalities to follow 
should be understood to hold for all $t \geq 2(\bar{\tau}+\nu)$. Next note that the time derivative of $\mathcal{P}(\lambda)=\frac{1}{2} \lambda^{2}$ satisfies

$$
\begin{aligned}
\dot{\mathcal{P}}(t)= & H(t) \lambda^{2}(t)+K_{*} \lambda(t) \int_{t-h_{\sigma(t)}-r(t)}^{t-h_{\sigma(t)}}[H(m) \lambda(m) \\
& \left.+K_{*}\left[\lambda\left(m-h_{\sigma(m)}\right)-\lambda(m-\tau(m))\right]\right] \mathrm{d} m \\
\leq & H(t) \lambda^{2}(t)+K_{*}|\lambda(t)| \int_{t-h_{\sigma(t)}-r(t)}^{t-h_{\sigma(t)}}\left[\nu^{\sharp}|\lambda(m)|\right. \\
& \left.+K_{*}\left(\left|\lambda\left(m-h_{\sigma(m)}\right)\right|+|\lambda(m-\tau(m))|\right)\right] \mathrm{d} m \\
\leq & \sqrt{2} K_{*} \sqrt{\mathcal{P}(\lambda(t))} \int_{t-h_{\sigma(t)}-r(t)}^{t-h_{\sigma(t)}}\left[K_{*}^{\sharp} \sqrt{\mathcal{P}(\lambda(m))}\right. \\
& +\sqrt{2} K_{*} \sqrt{\mathcal{P}\left(\lambda\left(m-h_{\sigma(m)}\right)\right)} \\
& \left.+\sqrt{2} K_{*} \sqrt{\mathcal{P}(\lambda(m-\tau(m)))}\right] \mathrm{d} m+2 H(t) \mathcal{P}(\lambda(t)) \\
\leq & 2 H(t) \mathcal{P}(\lambda(t))+M_{*} \sup _{\ell \in[t-2(\bar{\tau}+\nu), t]} \mathcal{P}(\lambda(\ell))
\end{aligned}
$$

along all solutions of (24), where $K_{*}^{\sharp}=\sqrt{2} \nu^{\sharp}, \nu^{\sharp}=1 / 8+$ $\left(1+2 e^{-\frac{\nu}{8}}+e^{-\frac{\nu}{4}}\right) K_{*}$, and $M_{*}=\sqrt{2} K_{*}\left(K_{*}^{\sharp}+2 \sqrt{2} K_{*}\right) \bar{r}$.

By integrating this inequality, we obtain

$$
\begin{aligned}
\mathcal{P}(\lambda(t)) \leq & e^{2 \int_{t-T}^{t} H(m) \mathrm{d} m} \mathcal{P}(\lambda(t-T)) \\
& +M_{*} \int_{t-T}^{t} e^{2 \int_{m}^{t} H(\ell) \mathrm{d} \ell} \sup _{\ell \in[m-2(\bar{\tau}+\nu), m]} \mathcal{P}(\lambda(\ell)) \mathrm{d} m \\
\leq & \left(e^{2 T H_{\star}}+M_{*} T e^{2 T \bar{H}}\right) S_{\lambda}(t)
\end{aligned}
$$

where $\bar{H}=\sup \{|H(\ell)|: \quad \ell \in \mathbb{R}\}$ and $S_{\lambda}(t)=$ $\sup _{\ell \in[t-T-2(\bar{\tau}+\nu), t]} \mathcal{P}(\lambda(\ell))$. Then [10, Lemma 1] ensures that (23) is uniformly globally exponentially stable to 0 if $e^{2 T H_{\star}}+2 M_{*} T e^{2 T \bar{H}} \in(0,1)$, i.e., if

$$
\bar{r}<\frac{1-e^{2 T H_{\star}}}{\sqrt{2} T K_{*}\left(\sqrt{2} \nu^{\sharp}+2 \sqrt{2} K_{*}\right) e^{2 T \bar{H}}} .
$$

Hence, (25) implies that Assumption 3 is satisfied, so the desired stabilizing feedback is given by (15)-(16)

\section{CONCLUSIONS}

We showed that stability of a system with a time-varying delay cannot be determined from the average value of the delay. This motivated our new representation of systems with time-varying delays, which we used to solve a stabilization problem with piecewise continuous delays. It would be worth comparing the performance of the controls from Section IV with those of [11]. We believe that our new representation eases the understanding of the effect of a time-varying delay and may be useful for solving other stability and control problems. We conjecture that extensions can be obtained for systems with several (pointwise and distributed) delays and with time-varying vector fields using the new representation we proposed. Nonlinear extensions are expected too.

\section{APpendix A: PROOF of LEMMA 3}

We assume that $\epsilon \in(0,1 / 2]$. Note that (7) gives

$$
\begin{aligned}
& g_{1}\left(t-\frac{3 \pi}{4}\right)=-\frac{\sqrt{2}}{2} g_{1}(t)-\frac{\sqrt{2}}{2} g_{2}(t) \\
& -\int_{t-\frac{3 \pi}{4}}^{t} \sin \left(t-m-\frac{3 \pi}{4}\right) u(m) \mathrm{d} m,
\end{aligned}
$$

by choosing $t_{*}=3 \pi / 4$. Setting $u=\epsilon y_{1}(t-3 \pi / 4)$ and $g=y$ in (A.1), we can write (8) as

$$
\left\{\begin{array}{l}
\dot{y}_{1}(t)=y_{2}(t) \\
\dot{y}_{2}(t)=-\left(1+\frac{\sqrt{2}}{2} \epsilon\right) y_{1}(t)-\frac{\sqrt{2}}{2} \epsilon y_{2}(t)-\epsilon^{2} \mathcal{J}(t)
\end{array}\right.
$$

where

$$
\mathcal{J}(t)=\int_{t-\frac{3 \pi}{4}}^{t} \sin \left(t-m-\frac{3 \pi}{4}\right) y_{1}\left(m-\frac{3 \pi}{4}\right) \mathrm{d} m .
$$

Using $\mathcal{A}(t, m)=t-m-\frac{3 \pi}{4}$ and noting that

$$
\begin{aligned}
& \mathcal{J}(t)=-\left(1+\frac{\sqrt{2}}{2}\right) y_{1}(t) \\
& +\int_{t-\frac{3 \pi}{4}}^{t} \sin (\mathcal{A}(t, m))\left[y_{1}\left(m-\frac{3 \pi}{4}\right)-y_{1}(t)\right] \mathrm{d} m \\
& =-\left(1+\frac{\sqrt{2}}{2}\right) y_{1}(t) \\
& -\int_{t-\frac{3 \pi}{4}}^{t} \sin (\mathcal{A}(t, m)) \int_{m-\frac{3 \pi}{4}}^{t} y_{2}(\ell) \mathrm{d} \ell \mathrm{d} m
\end{aligned}
$$

we obtain

$$
\left\{\begin{aligned}
\dot{y}_{1}(t)= & y_{2}(t) \\
\dot{y}_{2}(t)= & -q y_{1}(t)-\frac{\sqrt{2}}{2} \epsilon y_{2}(t) \\
& +\epsilon^{2} \int_{t-\frac{3 \pi}{4}}^{t} \sin (\mathcal{A}(t, m)) \int_{m-\frac{3 \pi}{4}}^{t} y_{2}(\ell) \mathrm{d} \ell \mathrm{d} m
\end{aligned}\right.
$$

where $q=1+\frac{\sqrt{2}}{2} \epsilon-\left(1+\frac{\sqrt{2}}{2}\right) \epsilon^{2}$ is positive, because $\epsilon \leq \frac{1}{2}$.

Let $Y=\left(y_{1}, y_{2}\right)$ and

$$
W\left(Y_{t}\right)=\frac{1}{2}\left[q y_{1}^{2}+y_{2}^{2}\right]+\frac{\sqrt{2}}{6 \pi} \epsilon \int_{t-\frac{3 \pi}{2}}^{t} \int_{m}^{t} y_{2}^{2}(s) \mathrm{d} s \mathrm{~d} m
$$

Simple calculations give

$$
\begin{aligned}
\dot{W}(t) \leq & \epsilon^{2} y_{2}(t) \int_{t-\frac{3 \pi}{4}}^{t} \sin (\mathcal{A}(t, m)) \int_{m-\frac{3 \pi}{4}}^{t} y_{2}(\ell) \mathrm{d} \ell \mathrm{d} m \\
& -\frac{\sqrt{2}}{6 \pi} \epsilon \int_{t-\frac{3 \pi}{2}}^{t} y_{2}^{2}(s) \mathrm{d} s+\frac{\sqrt{2}}{2 \pi} \frac{\pi}{2} \epsilon y_{2}^{2}(t)-\frac{\sqrt{2}}{2} \epsilon y_{2}^{2}(t) \\
\leq & -\frac{\sqrt{2}}{4} \epsilon y_{2}^{2}(t)-\frac{\sqrt{2}}{6 \pi} \epsilon \int_{t-\frac{3 \pi}{2}}^{t} y_{2}^{2}(s) \mathrm{d} s \\
& +\epsilon^{2}\left\{\left|y_{2}(t)\right| \int_{t-\frac{3 \pi}{4}}^{t} \int_{t-\frac{3 \pi}{2}}^{t}\left|y_{2}(\ell)\right| \mathrm{d} \ell \mathrm{d} m\right\} .
\end{aligned}
$$

By applying the triangle inequality to the quantity in curly braces and then applying Jensen's inequality, one can find a constant $\bar{\epsilon} \in(0,1 / 2]$ such that when $\epsilon \leq \bar{\epsilon}$, then

$$
\dot{W}(t) \leq-\frac{\sqrt{2}}{8} \epsilon y_{2}^{2}(t)-\frac{\sqrt{2}}{8 \pi} \epsilon \int_{t-\frac{3 \pi}{2}}^{t} y_{2}^{2}(s) \mathrm{d} s .
$$

This allows us to find small enough constants $\delta>0$ and $c_{0}>0$ such that the time derivative of $W^{\sharp}\left(Y_{t}\right)=W\left(Y_{t}\right)+$ $\delta y_{1}(t) y_{2}(t)$ along all solutions of (8) satisfies $\dot{W}^{\sharp}\left(Y_{t}\right) \leq$ $-c_{0} W^{\sharp}\left(Y_{t}\right)$ for large enough $t>0$, by writing

$$
\begin{aligned}
& \delta \epsilon y_{1}(t) y_{1}(t-3 \pi / 4) \leq \\
& \delta \epsilon\left[y_{1}^{2}(t)+\left\{\left|y_{1}(t)\right| \int_{t-3 \pi / 4}^{t}\left|y_{2}(m)\right| \mathrm{d} m\right\}\right],
\end{aligned}
$$

and applying the triangle inequality and then Jensen's inequality to the quantity in curly braces in (A.6). The global exponential stability of (8) for all $\epsilon \in(0, \bar{\epsilon})$ follows from the exponential decay of $W^{\sharp}$ and the positive definite quadratic upper and lower bounds on $W^{\sharp}$.

\section{ApPEndiX B: InSTABILITy OF SWitched System (9)}

The system (10) can be rewritten as:

$$
\left\{\begin{aligned}
\dot{x}_{1}(t)= & x_{2}(t) \\
\dot{x}_{2}(t)= & -x_{1}(t)+\frac{\epsilon}{2} x_{1}(t)+\frac{\epsilon}{2} x_{1}\left(t-\frac{3 \pi}{2}\right) \\
& +\epsilon\left(\frac{1}{2}-\sigma(t)\right)\left[x_{1}(t)-x_{1}\left(t-\frac{3 \pi}{2}\right)\right] .
\end{aligned}\right.
$$

From (7) and (10), we deduce that

$$
\begin{aligned}
& x_{1}\left(t-\frac{3 \pi}{2}\right)=x_{2}(t) \\
& -\epsilon \int_{t-\frac{3 \pi}{2}}^{t} \sin \left(t-m-\frac{3 \pi}{2}\right) \Delta(t, m) \mathrm{d} m,
\end{aligned}
$$


where $\Delta(t, m)=(1-\sigma(m)) x_{1}(m)+\sigma(m) x_{1}\left(m-\frac{3 \pi}{2}\right)$, by choosing $\left(g_{1}, g_{2}\right)=\left(x_{1}, x_{2}\right)$ in Lemma 2 . Then, we obtain

$$
\left\{\begin{aligned}
\dot{x}_{1}(t)= & x_{2}(t) \\
\dot{x}_{2}(t)= & \left(\frac{\epsilon}{2}-1\right) x_{1}(t)+\frac{\epsilon}{2} x_{2}(t) \\
& -\frac{\epsilon^{2}}{2} \int_{t-\frac{3 \pi}{2}}^{t} \sin \left(t-m-\frac{3 \pi}{2}\right) \Delta(t, m) \mathrm{d} m \\
& +\epsilon\left(\frac{1}{2}-\sigma(t)\right)\left[x_{1}(t)-x_{1}\left(t-\frac{3 \pi}{2}\right)\right] .
\end{aligned}\right.
$$

Let us define the function and operator

$$
\begin{aligned}
& \gamma(t)=\frac{1}{2 \nu} \int_{t-2 \nu}^{t} \int_{\ell}^{t}\left(\frac{1}{2}-\sigma(m)\right) \mathrm{d} m \mathrm{~d} \ell \\
& \text { and } \xi_{2}(t)=x_{2}(t)-\gamma(t) \epsilon\left[x_{1}(t)-x_{1}\left(t-\frac{3 \pi}{2}\right)\right],
\end{aligned}
$$

where $\nu$ is from the definition of $\sigma$. Then $|\gamma(t)| \leq \frac{\nu}{2}$ for all $t \in \mathbb{R}$. For almost all $t$, we can use the formula $\dot{\gamma}(t)=$ $\frac{1}{2}-\sigma(t)$ to get

$$
\left\{\begin{aligned}
\dot{x}_{1}(t)= & \xi_{2}(t)+\gamma(t) \epsilon\left[x_{1}(t)-x_{1}\left(t-\frac{3 \pi}{2}\right)\right] \\
\dot{\xi}_{2}(t)= & \left(\frac{\epsilon}{2}-1\right) x_{1}(t)+\frac{\epsilon}{2} x_{2}(t) \\
& -\frac{\epsilon^{2}}{2} \int_{t-\frac{3 \pi}{2}}^{t} \sin \left(t-m-\frac{3 \pi}{2}\right) \Delta(t, m) \mathrm{d} m \\
& -\gamma(t) \epsilon\left[x_{2}(t)-x_{2}\left(t-\frac{3 \pi}{2}\right)\right]
\end{aligned}\right.
$$

This gives

$$
\begin{aligned}
& \dot{\xi}_{2}(t)=\left(\frac{\epsilon}{2}-1\right) x_{1}(t)+\epsilon\left(\frac{1}{2}-\gamma(t)\right)\left(\xi_{2}(t)\right. \\
& \left.+\gamma(t) \epsilon\left[x_{1}(t)-x_{1}\left(t-\frac{3 \pi}{2}\right)\right]\right)+\gamma(t) \epsilon\left\{\xi_{2}\left(t-\frac{3 \pi}{2}\right)\right. \\
& \left.+\gamma\left(t-\frac{3 \pi}{2}\right) \epsilon\left[x_{1}\left(t-\frac{3 \pi}{2}\right)-x_{1}(t-3 \pi)\right]\right\} \\
& -\frac{\epsilon^{2}}{2} \int_{t-\frac{3 \pi}{2}}^{t} \sin \left(t-m-\frac{3 \pi}{2}\right) \Delta(t, m) \mathrm{d} m .
\end{aligned}
$$

It follows that all solutions of (10) satisfy

$$
\left\{\begin{array}{l}
\dot{x}_{1}(t)=\xi_{2}(t)+\kappa_{1}(t) \\
\dot{\xi}_{2}(t)=\left(\frac{\epsilon}{2}-1\right) x_{1}(t)+\frac{\epsilon}{2} \xi_{2}(t)+\kappa_{2}(t)
\end{array}\right.
$$

where $\kappa_{1}(t)=\gamma(t) \epsilon\left[x_{1}(t)-x_{1}\left(t-\frac{3 \pi}{2}\right)\right]$ and

$$
\begin{aligned}
\kappa_{2}(t)= & -\epsilon \gamma(t) \xi_{2}(t)+\epsilon\left(\frac{1}{2}-\gamma(t)\right) \\
& \times \gamma(t) \epsilon\left[x_{1}(t)-x_{1}\left(t-\frac{3 \pi}{2}\right)\right] \\
& +\gamma(t) \epsilon\left(\xi_{2}\left(t-\frac{3 \pi}{2}\right)+\gamma\left(t-\frac{3 \pi}{2}\right)\right. \\
& \left.\times \epsilon\left[x_{1}\left(t-\frac{3 \pi}{2}\right)-x_{1}(t-3 \pi)\right]\right) \\
& -\frac{\epsilon^{2}}{2} \int_{t-\frac{3 \pi}{2}}^{t} \sin \left(t-m-\frac{3 \pi}{2}\right) \Delta(t, m) \mathrm{d} m .
\end{aligned}
$$

Next observe that $S E+E^{\top} S=\frac{\epsilon}{2} S$, where

$$
S=\left[\begin{array}{cc}
\frac{1}{2}-\frac{\epsilon}{4} & -\frac{\epsilon}{8} \\
-\frac{\epsilon}{8} & \frac{1}{2}
\end{array}\right] \quad \text { and } \quad E=\left[\begin{array}{cc}
0 & 1 \\
\frac{\epsilon}{2}-1 & \frac{\epsilon}{2}
\end{array}\right]
$$

and where $S$ is symmetric and positive definite because $\epsilon \leq$ 1. Hence, with the choice $X=\left(x_{1}, \xi_{2}\right)$, the time derivative of $U(X)=X^{\top} S X$ along all solutions of (B.5) satisfies

$$
\begin{aligned}
\dot{U}(t)= & \frac{\epsilon}{2} U(X(t))+\left(1-\frac{\epsilon}{2}\right) x_{1}(t) \kappa_{1}(t) \\
& +\xi_{2}(t) \kappa_{2}(t)-\frac{\epsilon}{4} x_{1}(t) \kappa_{2}(t)-\frac{\epsilon}{4} \xi_{2}(t) \kappa_{1}(t) \\
\geq & \frac{\epsilon}{2} U(X(t))-\left\{\left|\xi_{2}(t)-\frac{\epsilon}{4} x_{1}(t)\right|\left|\kappa_{2}(t)\right|\right\} \\
& -\left\{\left|\left(1-\frac{\epsilon}{2}\right) x_{1}(t)-\frac{\epsilon}{4} \xi_{2}(t)\right|\left|\kappa_{1}(t)\right|\right\} .
\end{aligned}
$$

From (B.4), we deduce that for all $t \in \mathbb{R}$, we have

$$
\left|\kappa_{1}(t)\right| \leq \frac{\nu}{2} \epsilon\left|x_{1}(t)-x_{1}\left(t-\frac{3 \pi}{2}\right)\right| \text { and }
$$

$$
\begin{aligned}
& \left|\kappa_{2}(t)\right| \leq \frac{\nu \epsilon}{2}\left|\xi_{2}(t)\right|+\frac{\epsilon^{2} \nu(1+\nu)}{4}\left|x_{1}(t)-x_{1}\left(t-\frac{3 \pi}{2}\right)\right| \\
& +\frac{\nu^{2} \epsilon^{2}}{4}\left|x_{1}\left(t-\frac{3 \pi}{2}\right)-x_{1}(t-3 \pi)\right|+\frac{\nu \epsilon}{2}\left|\xi_{2}\left(t-\frac{3 \pi}{2}\right)\right| \\
& +\frac{\epsilon^{2}}{2} \int_{t-\frac{3 \pi}{2}}^{t}\left[\left|x_{1}(m)\right|+\left|x_{1}\left(m-\frac{3 \pi}{2}\right)\right|\right] \mathrm{d} m .
\end{aligned}
$$

Since there are positive constants $\bar{\kappa}$ and $T$ such that $\left|\kappa_{i}(t)\right| \leq$ $\left(\epsilon^{2}+\epsilon \nu\right) \bar{\kappa}|X|_{[t-T, t]}$ for all $t \geq 3 \pi$ with $\bar{\kappa}$ not depending on $\nu \in(0,1)$ or $\epsilon \in(0,1)$, we can apply the triangle inequality to the terms in curly braces and then Lemma 1 to (B.5) with $\mathcal{S}=U$ when $\nu$ and $\epsilon$ are sufficiently small positive constants. This provides an unbounded solution of (B.5) when $\nu$ and $\epsilon$ are sufficiently small positive constants. Then we can use our choice of $\xi_{2}$ from (B.4) to find an unbounded solution of (B.1).

Acknowledgment. The authors thank S.-I. Niculescu for indicating the enlightening references, notably [8].

\section{REFERENCES}

[1] N. Bekiaris-Liberis and M. Krstic. Compensation of time-varying input and state delays for nonlinear systems. ASME Journal of Dynamic Systems, Measurement, and Control, 134(1), 2012.

[2] D. Bresch-Pietri, F. Mazenc, and N. Petit. Robust compensation of a chattering time-varying input delay with jumps. Automatica, 92:225234, 2018.

[3] F. Bullo. Averaging and vibrational control of mechanical systems. SIAM J. Control Optim., 41(2):542-562, 2002.

[4] E. Fridman. Introduction to Time-Delay Systems, Analysis and Control. Birkhauser, Basel, 2014.

[5] J. Hale and S. Verduyn-Lunel. Averaging in infinite dimensions. Journal of Integral Equations and Applications, 2(4):463-494, 1990.

[6] B. Lehman and R. Bass. Extensions of averaging theory for power electronic systems. IEEE Trans. Power Electronics, 11(4):542-553, 1996.

[7] B. Lehman and P. Weibel. Averaging theory for delay difference equations with time-varying delays. SIAM Journal on Applied Mathematics, 59(4):1487-1506, 1999.

[8] J. Louisell. Delay differential systems with time-varying delay: New directions for stability theory. Kybernetica, 37(3):239-251, 2001.

[9] F. Mazenc, M. Malisoff, and S-I. Niculescu. Reduction model approach for linear time-varying systems with delays. IEEE Trans. Automatic Control, 59(8):2068-2081, 2014.

[10] F. Mazenc, M. Malisoff, and S-I. Niculescu. Stability and control design for time-varying systems with time-varying delays using a trajectory based approach. SIAM J. Control Optim., 55(1):533-556, 2017.

[11] F. Mazenc, M. Malisoff, and H. Ozbay. Stability and robustness analysis for switched systems with time-varying delays. SIAM J. Control Optim., 56(1):158-182, 2018.

[12] W. Michiels, V. Van Assche, and S-I. Niculescu. Stabilization of timedelay systems with a controlled time-varying delay and applications. IEEE Trans. Automatic Control, 50(4):493-504, 2005.

[13] S-I. Niculescu, C. de Souza, L. Dugard, and J-M. Dion. Robust exponential stability of uncertain systems with time-varying delays. IEEE Trans. Automatic Control, 43(5):743-748, 1998.

[14] S. Obuz, J. Klotz, R. Kamalapurkar, and W. Dixon. Unknown timevarying input delay compensation for uncertain nonlinear systems. Automatica, 76:222-229, 2017.

[15] J. Peuteman and D. Aeyels. Exponential stability of nonlinear timevarying differential equations and partial averaging. Mathematics of Control, Signals, and Systems, 15(1):42-70, 2002.

[16] E. Verriest. Robust stability of time varying systems with unknown bounded delays. In Proceedings of the 33rd IEEE Conference on Decision and Control, pages 417-422, Lake Buena Vista, FL, 1994.

[17] B. Zhou. Truncated Predictor Feedback for Time-Delay Systems. Springer-Verlag, Berlin, Germany, 2014.

[18] B. Zhou, Z. Lin, and G-R. Duan. Truncated predictor feedback for linear systems with long time-varying input delays. Automatica, 48:2387-2399, 2012. 